\title{
Right sneutrino with $\Delta L=2$ masses as nonthermal dark matter
}

\author{
Avirup Ghosh, ${ }^{1, *}$ Tanmoy Mondal, ${ }^{1,2, \dagger}$ and Biswarup Mukhopadhyaya ${ }^{1, \$}$ \\ ${ }^{1}$ Regional Centre for Accelerator-based Particle Physics, Harish-Chandra Research Institute, \\ HBNI, Chhatnag Road, Jhunsi, Allahabad-211 019, India \\ ${ }^{2}$ School of Physics, Korea Institute for Advanced Study, Seoul 130-722, Republic of Korea
}

(Received 28 November 2018; published 15 February 2019)

\begin{abstract}
We consider the minimal supersymmetric standard model (MSSM) with right-chiral neutrino superfields with Majorana masses, where the lightest right-handed sneutrino dominated scalars constitutes nonthermal dark matter (DM). The $\Delta L=2$ masses are subject to severe constraints coming from freeze-in relic density of such DM candidates as well as from sterile neutrino freeze-in. In addition, big bang nucleosynthesis and freeze-out of the next-to-lightest superparticle shrink the viable parameter space of such a scenario. We examine various $\Delta L=2$ mass terms for families other than that corresponding to the LSP sneutrino. $\Delta L=2$ masses are difficult to reconcile with a right-sneutrino DM, unless there is either (a) a hierarchy of about 3 orders of magnitudes among various supersymmetry-breaking mass parameters, or, (b) strong cancellation between the Higgsino mass and the trilinear supersymmetry breaking mass parameter for sneutrinos.
\end{abstract}

DOI: 10.1103/PhysRevD.99.035018

\section{INTRODUCTION}

While the search for the dark matter (DM) candidate(s) of our universe is on, one constantly feels the necessity of going beyond stereotypes in modeling physics beyond the standard model (SM) to accommodate the candidate particle(s). It is in this spirit that alternative candidates in small extensions of the minimal supersymmetric standard model (MSSM) have been explored. One example consists in scenarios with gravitino as warm DM [1-9]. Another, quite minimalistic, extension is to extend the MSSM with a right-chiral neutrino superfield for each family, and postulate that one right-sneutrino-dominated scalar is the DM candidate. Various cosmological as well as phenomenological implications of this scenario have already been explored [9-17].

The interaction of a sneutrino DM particle with all other MSSM fields is proportional to the very small neutrino Yukawa coupling. Consequently, this scenario almost always leads to a nonthermal DM, with a long-lived next-to-lightest SUSY particle (NLSP). Such a long-lived NLSP mostly survives till it decouples from the thermal

\footnotetext{
*avirupghosh@hri.res.in

tanmoymondal@hri.res.in

tbiswarup@hri.res.in
}

Published by the American Physical Society under the terms of the Creative Commons Attribution 4.0 International license. Further distribution of this work must maintain attribution to the author(s) and the published article's title, journal citation, and DOI. Funded by SCOAP ${ }^{3}$. bath, and decays to the DM candidate thereafter. Such scenarios have been often explored by assuming that neutrinos have just Dirac masses, in which cases their Yukawa coupling strengths are $\mathcal{O}\left(10^{-13}\right)$ [13,18-25].

Some additional issues become important if neutrinos have Majorana masses as well. This will require the righthanded neutrino fields to have $\Delta L=2$ mass terms. The Type-I seesaw mechanism works here, thus requiring somewhat higher neutrino Yukawa couplings. This, however, entails the process of freeze-in of right sneutrinos while the NLSP (a stau, for example) is yet to decouple. Studies including those on similar non-SUSY theories have shown that such freeze-in contribution to the relic density impose rather strong constraints on the corresponding model parameters [26-29]. In MSSM with right sneutrino DM and Dirac masses, too, this effect is small but nonnegligable and it constrains the associated parameter space [10,23-25]. With $\Delta L=2$ mass terms present, however, the seesaw formula allows bigger Yukawa couplings, and thus the constraints tighten. For a degenerate light neutrino spectrum around $0.1 \mathrm{eV}$ the limit on Majorana masses and Yukawa coupling was studied in [30]. These observations were restricted to specific scenarios where all relevant SUSY-breaking parameters were taken to be around the electroweak scale (i.e., $100 \mathrm{GeV}$ ). In [31] it was shown that the near degeneracy among the NLSP and LSP decreases the relic density due to inverse decay of LSP.

The present study is more general, and takes into account wider ranges of mass scales as well the interplay of various SUSY-breaking parameters. Unlike most approaches we allow a hierarchy of SUSY-breaking mass parameters. 
The freeze-in of right-sneutrino DM from the decay of heavier superparticles will be affected by the (small but nonvanishing) left-sneutrino component in the DM and hence the deciding factor is the sneutrino mixing angle, which essentially depends on the off-diagonal part of the sneutrino mass matrix and is tightly constrained. One has to also fit the neutrino mass and mixing patterns [32], and, quite seriously, the potential contribution of longlived sterile neutrinos generated via the DodelsonWidrow mechanism [33-40]. Thus the $\Delta L=2$ masses, especially those corresponding to the families other than the one the DM belongs to, get seriously restricted, especially in view of the present limit on the dark matter relic density by the PLANCK collaboration [41]. The way such restrictions arise is investigated in the present paper.

There can be several scales associated with the $\Delta L=2$ masses, consistent with the Type-I seesaw mechanism. The commonly known GUT-scale seesaw will not work for a case where nonthermal DM candidates are sought, as that would entail Yukawa couplings large enough for them to thermalize. We find that, Majorana masses in the electroweak scale, too, allow Yukawa couplings consistent with freeze-in rate, if a suppression in left-right mixing occurs via diagonal SUSY breaking terms that are several orders of magnitude larger than the off-diagonal ones. Though the situation is marginally better for $\mathcal{O}(1) \mathrm{GeV}$ Majorana masses, a fine-tuned cancellation between the soft-and F-terms is required there as well. The scenario with all the three Majorana masses ranging from $500 \mathrm{MeV}$ down to a few tens of $\mathrm{keV}$ is constrained by light element abundance. When all three masses are in $\mathrm{keV}$ scale, they all become warm dark matter, a scenario ruled out already due to overproduction of the warm $\mathrm{keV}$ scale dark matter via Dodelson-Widrow mechanism. We may have one $\Delta L=2$ mass in the $\mathrm{eV}$ scale [42], as considered in [30], but such a situation brings in severe cosmological constraints $[41,43]$. We establish freeze-in of right-sneutrino DM to be a major constraining factor with $\Delta L=2$ masses. In such a case, not only the NLSP but also the rest of the MSSM spectrum contributes to freeze-in. This constraint is thus largely applicable to scenarios including various NLSP candidates. We focus on the stau and neutralino NLSP in particular.

Our paper have been organized as follows. In Sec. II we discuss the model considered by us and the related constrains coming from freeze-in, NLSP freeze-out, low mass sterile neutrinos and big bang nucleosynthesis (BBN). Section III has been devoted to the discussions of the analysis strategy followed by us in determining the allowed parameter space still allowed from the constraints mentioned in Sec. II. In Sec. IV we have shown how the constraints mentioned already can severely restrict the parameter space of such a scenario following the strategy mentioned. We summarize and conclude in Sec. V. Finally the formulas used by us have been tabulated in Appendices $\mathrm{A}$ and $\mathrm{B}$.

\section{THE MODEL AND CONSTRAINTS}

We start by outlining the salient features of a right sneutrino DM scenario with $\Delta L=2$ masses, which warrant a fresh study of the constraints at focus. We consider the MSSM scenario augmented with three right chiral neutrino superfields $\left(\hat{N}_{R}\right)$, which possess $\Delta L=2$ mass terms. Hence the superpotential gets extended to the form [44-46]

$$
\mathcal{W}=\mathcal{W}_{\mathrm{MSSM}}+Y_{\nu}^{i j} \hat{H}_{u} \hat{L}^{i} \hat{N}_{R}^{j}+\frac{1}{2} M_{N}^{i j} \hat{N}_{R}^{i} \hat{N}_{R}^{j},
$$

where $\hat{H}_{u}$ is the Higgs doublet that couples to up-type quarks, $\hat{L}$ is left-chiral SU(2) doublet lepton superfields and $\hat{N}_{R}$ is a right-handed neutrino superfield. While $Y_{\nu}$ is the neutrino Yukawa coupling matrix, $M_{N}$ is the $\Delta L=2$ mass matrix for the heavy neutrinos, assumed, as already stated, to be diagonal, since basis rotations in the right-handed neutrino sector is unlikely to affect our main conclusions.

Since we are not assuming any high-scale mechanism of SUSY breaking we need to add all allowed SUSY-breaking terms phenomenologically. The relevant soft terms in this case are

$$
\begin{aligned}
\mathcal{L}_{\text {soft }}= & \mathcal{L}_{\text {soft }}^{\mathrm{MSSM}}-m_{N}^{2 i j} \tilde{N}_{R}^{* i} \tilde{N}_{R}^{j}+\left(m_{B}^{2 i j} \tilde{N}_{R}^{i} \tilde{N}_{R}^{j}\right. \\
& \left.-T_{\nu}^{i j} h_{u} \tilde{L}^{i} \tilde{N}_{R}^{j}+\text { H.c. }\right)
\end{aligned}
$$

where $m_{N}\left(m_{B}\right)$ corresponds to $\Delta L=0$ (2) SUSY-breaking sneutrino masses and $T_{\nu}$ is the coefficient of the trilinear SUSY-breaking term. The left-right mixing in the sneutrino sector occurs, via the soft terms proportional to $T_{\nu}$, and the F-terms proportional to the Higgsino mass parameter $(\mu)$, when $h_{u}\left(h_{d}\right)$ acquires the vacuum expectation values $v_{u}\left(v_{d}\right)$. In addition to $m_{N}$ and $m_{B}$, the F-term masses proportional to $M_{N}$ adds to the right-handed sneutrino masses. In our case, one requires $M_{N}$ not to exceed this SUSY-breaking scale, so that a right sneutrino may behave as a DM candidate.

To study the sneutrino mass terms and phenomenology of sneutrinos it is convenient to introduce the real fields, $\left(\tilde{\nu}_{1}^{i}, \tilde{N}_{1}^{i}, \tilde{\nu}_{2}^{i}, \tilde{N}_{2}^{i}\right)$ defined as follows [30,31,46]:

$$
\tilde{\nu}_{L}^{i}=\frac{1}{\sqrt{2}}\left(\tilde{\nu}_{1}^{i}+i \tilde{\nu}_{2}^{i}\right), \quad \tilde{N}_{R}^{i}=\frac{1}{\sqrt{2}}\left(\tilde{N}_{1}^{i}+i \tilde{N}_{2}^{i}\right),
$$

In the basis constituted by $C P$-even $\left(\tilde{\nu}_{1}, \tilde{N}_{1}\right)$ and $C P$-odd $\left(\tilde{\nu}_{2}, \tilde{N}_{2}\right)$ real sneutrino fields, assuming no $C P$-violation in the SUSY sector the sneutrino mass matrix takes the blockdiagonal form 


$$
M_{\tilde{\nu}}=\left[\begin{array}{cccc}
m_{L L}^{2} & m_{R L}^{2}+m_{D} M_{N} & 0 & 0 \\
m_{R L}^{2 T}+M_{N}^{T} m_{D}^{T} & m_{R R}^{2}-m_{B}^{2} & 0 & 0 \\
0 & 0 & m_{L L}^{2} & m_{R L}^{2}-m_{D} M_{N} \\
0 & 0 & m_{R L}^{2 T}-M_{N}^{T} m_{D}^{T} & m_{R R}^{2}+m_{B}^{2}
\end{array}\right] .
$$

where $m_{L L}^{2}=m_{\tilde{l}_{L}}^{2}+\frac{v_{u}^{2}}{2}\left|Y_{\nu}\right|^{2}+\frac{m_{z}^{2}}{2} \cos 2 \beta, m_{R L}^{2}=-\mu^{*} \frac{v_{d}}{\sqrt{2}} Y_{\nu}+$ $\frac{v_{u}}{\sqrt{2}} T_{\nu}$ and $m_{R R}^{2}=M_{N}^{2}+m_{N}^{2}+\frac{v_{u}^{2}}{2}\left|Y_{\nu}\right|^{2}$ are the $3 \times 3$ mass matrices in the flavor basis, $m_{D}=Y_{\nu} \frac{v_{u}}{\sqrt{2}}$ is the Dirac mass matrix for neutrinos and $m_{\tilde{l}_{L}}$ is the soft mass term for the left-chiral slepton doublet $\tilde{L}$ (suppressing generation indices). $T_{\nu}$ will be parametrised as $T_{\nu}=A_{\nu} Y_{\nu}, A_{\nu}$ being a mass-scale related to the scale of SUSY-breaking.

Each of the $6 \times 6$ blocks of the mass matrix in Eq. (4) can be diagonalized by an unitary transformation, which mixes the right-chiral and left-chiral sneutrinos (separately in both $C P$-even and $C P$-odd sectors). The $3 \times 3$ mixing matrix between left-chiral and right-chiral sneutrinos is given by (when the off-diagonal elements of each of the two blocks are much smaller than corresponding diagonal elements),

$$
\Theta_{\tilde{\nu}} \approx 2\left[m_{L L}^{2}-\left(m_{R R}^{2} \mp m_{B}^{2}\right)\right]^{-1}\left[\left(m_{R L}^{2} \pm m_{D} M_{N}\right)\right]^{T},
$$

which is a multiplication of two matrices where the upper (lower) sign corresponds to mixing in the $C P$-even ( $C P$-odd) sector.

Since there is no mixing between the $C P$-even and $C P$ odd sectors of the sneutrinos, both the lightest $C P$-even as well as the lightest $C P$-odd snutrino will be dark matter in this scenario. ${ }^{1} \mathrm{We}$ are considering both $(C P$ even as well as $C P$-odd) lightest sneutrinos to be dominantly right-handed (i.e., small left-right mixing angle $\left(\Theta_{\tilde{\nu}}\right)_{1 j}$ with $\left.\mathrm{j}=1,2,3\right)$ with masses

$M_{\tilde{\nu}_{\mathrm{DM}}^{1}\left(\tilde{\nu}_{\mathrm{DM}}^{2}\right)} \simeq \sqrt{m_{R R}^{2} \mp m_{B}^{2}}=\sqrt{M_{N}^{2}+m_{N}^{2}+\frac{v_{u}^{2}}{2}\left|Y_{\nu}\right|^{2} \mp m_{B}^{2}}$,

where the upper(lower) sign is for the $C P$-even(odd) dark matter $\tilde{\nu}_{\mathrm{DM}}^{1}\left(\tilde{\nu}_{\mathrm{DM}}^{2}\right)$.

The right-handed sneutrinos $\left(\tilde{\nu}_{R}\right)$ being SM gauge singlets, their only interactions are governed by the mixing (denoted by $\Theta_{\tilde{\nu}}$ ) with the left-handed sneutrinos $\tilde{\nu}_{L}$.

\footnotetext{
${ }^{1}$ Since the only possible decay of the $C P$-odd lightest sneutrino is into the $C P$-even one and the corresponding amplitude is suppressed by the squares of the neutrino Yukawa coupling, the lifetime of this $C P$-odd lightest sneutrino will always be larger than the age of the Universe. We have ensured this lifetime to be typically of order $\simeq 10^{27} \mathrm{sec}$ with proper choice of parameters.
}

Pertaining to such small interaction strength, LSP $\tilde{\nu}_{R} \mathrm{~S}$ never reaches thermal equilibrium and are produced from the decay of heavier superparticles (left-handed sleptons $\tilde{l}_{L}$, left-handed sneutrinos $\tilde{\nu}_{L}$, neutralinos $\chi_{i}^{0}$, and charginos $\left.\chi_{i}^{ \pm}\right)$. Such production mechanism of nonthermal DM particles is called freeze-in [47-58].

The yield of a DM particle produced via freeze-in is given by [49],

$Y_{\tilde{\nu}_{\mathrm{DM}}^{i}}=\frac{n_{\tilde{\nu}_{\mathrm{DM}}^{i}}}{s} \simeq \frac{45}{1.66 \times 4 \pi^{4}} \frac{M_{\mathrm{P}}}{g_{*}^{s} \sqrt{g_{*}^{\rho}}} \sum_{\text {allA }} \frac{g_{A} \Gamma_{A}^{i}}{m_{A}^{2}} \int_{x=0}^{x=\infty} K_{1}(x) x^{3} d x$,

where $n_{\tilde{\nu}_{\mathrm{DM}}^{i}}$ and $s$ being the number density of the DM candidate and entropy density of the universe at the time of freeze-in respectively. Furthermore, $m_{A}$ and $g_{A}$ are the mass and degrees of freedom (d.o.f.) of the decaying superparticle A, whereas $\Gamma_{A}^{i}$ is its decay width to the $\tilde{\nu}_{\mathrm{DM}}^{i}$. All the relevant decay amplitudes and decay widths are given in Appendices A and B. The ratio $\frac{m_{A}}{T}$ is define as $x$ and $g_{*}^{s, \rho}$ are the number of d.o.f. at the time of freeze-in, i.e., $T \simeq m_{A}$ for entropy $s$ and energy density $\rho$ respectively.

The relic density via freeze-in is given by [59],

$$
\Omega_{\mathrm{FI}} h^{2}=2.755 \times 10^{8} \sum_{i} M_{\tilde{\nu}_{\mathrm{DM}}^{i}} Y_{\tilde{\nu}_{\mathrm{DM}}^{i}},
$$

where $M_{\tilde{\nu}_{\mathrm{DM}}^{i}}$ is the mass of sneutrino dark matter and the index $i$ takes care of both the $C P$-even and $C P$-odd sneutrino mass eigenstates. Using the values of $M_{\mathrm{Pl}}=10^{19} \mathrm{GeV}, g_{*}^{s} \simeq g_{*}^{\rho} \simeq 100$ [60] in equation (7), the freeze-in relic density of $\tilde{\nu}_{\mathrm{DM}}$, denoted by $\Omega_{\mathrm{FI}}$ in Eq. (8) can be estimated as,

$$
\Omega_{\mathrm{FI}} h^{2} \simeq 1.92 \times 10^{23} \sum_{i} M_{\tilde{\nu}_{\mathrm{DM}}^{i}} \frac{\Gamma_{\text {tot }}^{i}}{m_{\text {susy }}^{2}}
$$

where $\Gamma_{\text {tot }}^{i}=\sum_{\text {all } A} g_{A} \Gamma_{A}^{i} \int_{x=0}^{x=\infty} K_{1}(x) x^{3} d x$ with $g_{A}$ being the appropriate d.o.f. for each of the decaying particles. $M_{\tilde{\nu}_{\mathrm{DM}}^{i}}$ is the mass of $\tilde{\nu}_{\mathrm{DM}}^{i}$ and $m_{\text {susy }}$ is the generic value of the SUSY-breaking mass parameters in the electroweak sector of the MSSM part of the spectrum.

Over and above freeze-in, the post freeze-out decay of next-to-lightest superparticle(NLSP) also gives rise to some amount of DM relic, given by $[47,48]$ 
$\Omega_{\mathrm{FO}} h^{2}=\left(\sum_{i} \mathrm{BR}\left(\mathrm{NLSP} \rightarrow \tilde{\nu}_{\mathrm{DM}}^{i}+\mathrm{SM}\right) \frac{M_{\tilde{\nu}_{\mathrm{DM}}^{i}}}{M_{\mathrm{NLSP}}}\right) \Omega_{\mathrm{NLSP}} h^{2}$,

In our analysis we have considered the possibilities of dominantly right-handed stau $\left(\tilde{\tau}_{R}\right)$ and bino or Higgsino dominated neutralino $\left(\chi_{1}^{0}\right)$ being the NLSP. In any case the total relic density is determined by,

$$
\Omega_{\tilde{\nu}_{\mathrm{DM}}} h^{2}=\Omega_{\mathrm{FI}} h^{2}+\Omega_{\mathrm{FO}} h^{2} .
$$

While carrying out our analysis we have ensured that our chosen parameter region do satisfy the following constraints.

(i) PLANCK collaboration sets an upper limit on the DM relic density [41],

$$
\Omega_{\mathrm{CDM}} h^{2}=0.1199 \pm 0.0027 .
$$

which we have respected through out our analysis in determining the allowed parameter space of the model.

(ii) The usual constraints on neutrino masses and mixing angles, mostly derived from oscillation data, have been satisfied. In addition, since neutrino Yukawa couplings $\left(Y_{\nu}\right)$ and neutrino Majorana masses $\left(M_{N}\right)$ play a major role in determining the left-right mixing angles $\left(\Theta_{\tilde{\nu}}\right)$, all the associated bounds (BBN constraints on heavy neutrino decays, $\mathrm{X}$-ray and
Gamma-ray observations, DW production, Lyman$\alpha$ forest) on the light sterile neutrinos have been taken into account $[33,37,61-67]$.

(iii) The only possible decays of frozen-out NLSP into $\tilde{\nu}_{\mathrm{DM}}$ being suppressed by left-right mixing $\Theta_{\tilde{\nu} 1 j}$, (where $\mathrm{j}=1,2,3$ ) the NLSPs are fairly long-lived and can potentially affect the standard BBN predictions $[13,43,68-72]$. We have taken note of these constraints through out our analysis by assuming $\tau_{\mathrm{NLSP}} \leq 100 \mathrm{sec}$. It is difficult to accommodate a bino dominated neutralino as NLSP in such scenarios since the late decay of such a neutralino gives rise to an unacceptably large sneutrino relic density. One's best bet thus is either a Higgsino dominated neutralino or a dominantly right-handed stau to be the NLSP. In the range $M_{\mathrm{NLSP}} \simeq 300-900 \mathrm{GeV}$ such NLSP can only give rise to $1 \%$ of the total relic density (for $M_{\tilde{\nu}_{\mathrm{DM}}} \simeq 100 \mathrm{GeV}$ ) and hence one can safely ignore their contribution and concentrate in the freeze-in contribution solely.

(iv) The mass of lightest Higgs emerging from the spectrum calculation using SPHENO [73] is kept in the range $123 \mathrm{GeV}<m_{h^{0}}<128 \mathrm{GeV}[74,75]$. The stop mass parameters $\left(m_{\tilde{t}_{1,2}}\right)$ and trilinear $\left(A_{t}\right)$ couplings have been fixed at such values as to ensure the observed value of $m_{h^{0}}$ (see Table I), which does not affect our arguments presented in this paper. The mass window have been kept a little lenient, in order to account for various theoretical uncertainties.

TABLE I. Input parameters and their ranges scanned in our analysis are tabulated here. The neutrino oscillation parameters, $\left(m_{N}\right)^{H},\left(m_{B}\right)^{H},\left(M_{N}\right)_{11}$ and $\left(m_{B}\right)_{11}$ have been kept fixed as they have very little or no effect on the analysis carried out. The Majorana masses $\left(M_{N}\right)^{H}$ and other supersymmetric parameters like $\left(m_{N}\right)_{11}, \mu, \tan \beta$ and $A_{\nu}$ have been varied such that one can satisfy the necessary constraints on the freeze-in relic density (see equations. (14) and (19) respectively).

\begin{tabular}{lcc}
\hline \hline Parameter & Ranges $\left(m_{\text {susy }} \simeq 1 \mathrm{TeV}\right)$ & Ranges $\left(m_{\text {susy }} \simeq 100 \mathrm{TeV}\right)$ \\
\hline$\left\{\theta_{12}, \theta_{23}, \theta_{13}\right\}$ & $\left\{33.62^{\circ}, 47.2^{\circ}, 8.54^{\circ}\right\}$ & $\left\{33.62^{\circ}, 47.2^{\circ}, 8.54^{\circ}\right\}$ \\
$\left\{\delta_{C P}, \alpha_{1}, \alpha_{2}\right\}$ & $\left\{\frac{3 \pi}{2}, \frac{\pi}{4}, \frac{\pi}{4}\right\}$ & $\left\{\frac{3 \pi}{2}, \frac{\pi}{4}, \frac{\pi}{4}\right\}$ \\
$\left(M_{N}\right)_{11}$ & $10 \mathrm{keV}$ & $10 \mathrm{keV}$ \\
$M_{N}^{H}$ & $10 \mathrm{keV}-50 \mathrm{keV}, 500 \mathrm{MeV}-\mathcal{O}(10 \mathrm{GeV})$ & $\mathcal{O}(10 \mathrm{GeV})-200 \mathrm{GeV}$ \\
$\left(m_{N}\right)_{11}$ & $20 \mathrm{GeV}-400 \mathrm{GeV}$ & $20 \mathrm{GeV}-200 \mathrm{GeV}$ \\
$\left(m_{B}\right)_{11}$ & $10 \mathrm{GeV}$ & $10 \mathrm{GeV}$ \\
$\mu$ & $500 \mathrm{GeV}-1.5 \mathrm{TeV}$ & $500 \mathrm{GeV}-1.5 \mathrm{TeV}$ \\
$\tan \beta$ & 10.5 & 3.5 \\
$A_{\nu}$ & $20 \mathrm{GeV}-400 \mathrm{GeV}$ & $20 \mathrm{GeV}-400 \mathrm{GeV}$ \\
$\left(m_{N}\right)^{H}$ & $1 \mathrm{TeV}$ & $1 \mathrm{TeV}$ \\
$\left(m_{B}\right)^{H}$ & $175 \mathrm{GeV}$ & $175 \mathrm{GeV}$ \\
$m_{\tilde{t}_{1}}$ & $1225 \mathrm{GeV}$ & $500 \mathrm{TeV}$ \\
$m_{\tilde{t}_{2}}$ & $1725 \mathrm{GeV}$ & $502.4 \mathrm{TeV}$ \\
$A_{t}$ & $3890 \mathrm{GeV}$ & $2089 \mathrm{TeV}$ \\
$m_{h^{0}}$ & $125.62 \mathrm{GeV}$ & $124.77 \mathrm{GeV}$ \\
$m_{A^{0}}$ & $2 \mathrm{TeV}$ & $3640 \mathrm{TeV}$ \\
$m_{\tilde{q}_{L, R}}$ & $3 \mathrm{TeV}$ & $600 \mathrm{TeV}$ \\
$m_{\tilde{g}}$ & $3 \mathrm{TeV}$ & $600 \mathrm{TeV}$ \\
\hline \hline
\end{tabular}


Moreover, the consistency of lightest Higgs couplings with LHC data [76,77] have been ensured with the help of LILITH [78] and HigGsBounds [79].

(v) The PMNS-driven contributions to FCNC processes like $\mu \rightarrow e \gamma, \tau \rightarrow \mu \gamma$ etc. are within the current limits [80].

\section{STRATEGY OF OUR ANALYSIS}

We summarize next the methodology adopted in obtaining the new results contained in this paper. The sneutrino relic density via freeze-in depends on the decay width of heavier superparticles into it. Hence the corresponding decay widths and the left-right mixing angles $\Theta_{\tilde{\nu} 1 j}$ (with $\mathrm{j}=1,2,3$ ) are highly constrained by the PLANCK limit of relic abundance. ${ }^{2}$ For a dominantly right-handed sneutrino dark matter, all the mixing angles $\Theta_{\tilde{\nu} 1 j}$ depend on $m_{\text {susy }} \approx m_{\tilde{l}_{L}}$. The bino $\left(m_{\tilde{B}}\right)$ and wino $\left(m_{\tilde{W}^{3}}\right)$ mass parameters are also in the same value for simplicity, and henceforth $m_{\tilde{l}_{L}}, m_{\tilde{B}}$ and $m_{\tilde{W}^{3}}$ will collectively be denoted as $m_{\text {susy }}$.

We have considered two representative cases, with $m_{\text {susy }} \simeq 1 \mathrm{TeV}$ (which is accessible at the LHC) and $m_{\text {susy }} \simeq \mathcal{O}(100) \mathrm{TeV}$ (inaccessible at the colliders). For any $m_{\text {susy }}$, Eqs. (5) and (9) allow one to translate the limits on $\Theta_{\tilde{\nu} 1 j}$ into constraints on the parameters $A_{\nu}, \mu, \tan \beta, Y_{\nu}$ and, last but not the least, on $m_{R R}$ and $m_{B}$ (see Sec. IV for details).

In order to study the dependence of relic density on these parameters, we have varied them in several ranges quoted in Table I. The best fit values for the neutrino oscillation parameters have been taken from [32]. The Majorana phases $\left(\alpha_{1}, \alpha_{2}\right)$ have been set to $\frac{\pi}{4}$ since variation in them does not change the order of magnitude of $Y_{\nu}$, which is all important for the scenario under consideration. $\left(M_{N}\right)_{11}$, $\left(m_{N}\right)_{11}\left[\left(m_{B}\right)_{11}\right]$ are respectively the Majorana mass, $\Delta L=$ 0 (2) SUSY-breaking masses for the LSP sneutrino, while $M_{N}^{H},\left(m_{N}\right)^{H}\left[\left(m_{B}\right)^{H}\right]$ are the corresponding parameters for the remaining two heavier right-handed sneutrinos. One should note that in both of the aforementioned cases of $m_{\text {susy }}$, we have used $\left(m_{B}\right)_{11} \ll\left(m_{N}\right)_{11}$ in order to ensure $\tau_{\tilde{\nu}_{\mathrm{DM}}^{2}} \geq 10^{27}$ sec. For $m_{\text {susy }} \simeq 1 \mathrm{TeV}$, the region $50 \mathrm{keV}<$ $M_{N}^{H}<500 \mathrm{MeV}$ has been left out, since such values cause the heavy neutrinos to decay after $100 \mathrm{sec}$. [34], thereby disturbing standard BBN predictions. Overall, while our analysis is insensitive to the choices of $\left(m_{N}\right)^{H}$ and $\left(m_{B}\right)^{H}$, the benchmark values (shown in Table I) are chosen to ensure that the heavier sneutrino mass eigenstates are heavier than the NLSP and decay before BBN. The same

\footnotetext{
${ }^{2}$ Henceforth we will denote all the mixing angles of $\tilde{\nu}$ LSP collectively as $\Theta_{\tilde{\nu} 1 j}$ without mentioning the values $\mathrm{j}$ can acquire since our arguments apply to all possible $j$ values. When we refer to any particular mixing angle the specific value of $\mathrm{j}$ will be mentioned.
}

argument applies to the parameters $\left(m_{\tilde{t}_{1,2}}, A_{t}, m_{\tilde{q}_{L, R}}, m_{\tilde{g}}\right.$, $m_{A^{0}}$ ) related to strongly interacting sector and Higgs sector of the model. All these parameters have been kept fixed through out the analysis at the values quoted in Table I although our conclusion is not specific to such values.

Higgsino freeze-in: The situation is different when the Higgsino component in $\chi_{i}^{0}(i=1, \ldots 4)$ decays into $\tilde{\nu}_{\mathrm{DM}}^{i}$ while the former is in the equilibrium with the thermal bath. The decay amplitude in this case depends solely on $Y_{\nu}$. If the right sneutrino LSP is of the same family as that of the lightest active neutrino, then the Higgsino decay width to the LSP sneutrino is solely determined by the lightest neutrino Yukawa couplings $\left(Y_{\nu}\right)_{1 i},(i=1,2,3)$, assuming normal hierarchy $(\mathrm{NH})$ of neutrino masses. ${ }^{3}$ We have found that for $\left(Y_{\nu}\right)_{1 i} \simeq 10^{-12}$ the Higgsino decay saturates the sneutrino relic abundance. Since the lightest active neutrino mass is a free parameter in the hierarchical scenario, it can be tuned to control the contribution of any Higgsinodominated neutralino to relic abundance. Using this freedom if we reduce these Yukawa couplings to $\mathcal{O}\left(10^{-14}\right)$ or less, the Higgsino contribution is even lesser. In order to look for the effect of other parameters on the relic density we have been a little indulgent in our choice of $\left(Y_{\nu}\right)_{1 i}$. Throughout our analysis, these Yukawa couplings corresponding to the flavor eigenstates dominating the lightest mass eigenstate is thus set to $\mathcal{O}\left(10^{-13}\right)$ thus yielding the lightest neutrino mass $m_{1} \simeq 10^{-8} \mathrm{eV}$ for a $10 \mathrm{keV}$ Majorana mass. The freeze-in of Higgsinos in this case contributes about $1 \%$ of the total relic of $\tilde{\nu}_{\mathrm{DM}}$. This choice considerably simplifies our analysis. The more general mixing scenario multiplies the number of free parameters but does not affect the constraint on the Majorana mass of the lightest neutrino. The Yukawa couplings for other neutrinos are dictated by the Majorana mass(es) and neutrino oscillation data. These couplings can be considerably large compared to the pure Dirac case. One can use the Casas-Ibarra parametrization $[81]^{4}$ to write,

\footnotetext{
${ }^{3}$ For inverted hierarchy (IH), the conclusions are not very different if the sneutrino associated with the lightest active neutrino is the DM candidate. For the quasi- degenerate scenario, the lightest neutrino mass is bound to be $\simeq 10^{-2} \mathrm{eV}$. In order to prevent Higgsino decays from overproducing sneutrino dark matter via Yukawa interaction, one has to lower the Majorana mass corresponding to the lightest neutrino eigenstate. One thus finds oneself pushed down to $\mathrm{eV}$-scale value for this Majorana mass. Such a situation has already been mentioned in [30]; however, recent limits from $\mathrm{BBN}$ and recombination $[41,43]$ strongly restricts this scenario.

${ }^{4}$ Since for our present analysis only the order of magnitude of the Dirac Yukawa matrix elements matters, the Casas- Ibarra parametrization helps us fix the Yukawa matrix conveniently which satisfies neutrino oscillation data by construction, without any random sampling.
} 


$$
Y_{\nu}=\frac{-i \sqrt{2}}{v_{u}} \sqrt{M_{N}} R \sqrt{m_{\nu}^{\text {diag }}} U_{\mathrm{PMNS}}^{\dagger}
$$

where, $R$ is a complex orthogonal matrix, $m_{\nu}^{\text {diag }}$ is the diagonalized light-neutrino mass matrix (whose elements are determined by $\Delta m_{\odot}^{2}$ and $\Delta m_{\oplus}^{2}$ ) and $U_{\text {PMNS }}$ is the diagonalizing matrix. Since one light neutrino is effectively massless, we have parametrized $R$ as [82]

$$
R=\left[\begin{array}{ccc}
1 & 0 & 0 \\
0 & \cos \omega & \sin \omega \\
0 & -\sin \omega & \cos \omega
\end{array}\right],
$$

where a nonvanishing $R_{11}$ with appropriate $m_{\nu}^{\text {diag }}$ reflects the postulated hierarchy. We use $\omega=i$.

\section{RESULTS}

As has been mentioned in Sec. III, very high $\Delta L=2$ neutrino masses lead to Yukawa couplings that are inadmissible from freeze-in constraints. We consider two representative values of $m_{\text {susy }}$, namely $1 \mathrm{TeV}$ and $100 \mathrm{TeV}$. Both of these ranges are consistent with freeze-in constraints.

\section{A. Results for $m_{\text {susy }} \simeq 1 \mathrm{TeV}$}

In this scenario with all MSSM superparticles in the TeV scale, Eq. (9) implies [41],

$$
\sum_{i} M_{\tilde{\nu}_{\mathrm{DM}}^{i}} \Gamma_{\tilde{\nu}_{\mathrm{DM}}^{i}} \lesssim 5.2 \times 10^{-19} \mathrm{GeV}^{2} .
$$

The sfermions give the dominant contribution to the decay width compared to the contributions from bino and winos since it is enhanced by a phase-space factor of $\frac{m_{\text {susy }}^{2}}{m_{\mathrm{EW}}^{2}}$ [e.g., compare (A1e)-(A1f)]. Thus for DM masses around $100 \mathrm{GeV}$, Eq. (14) leads to $\left(\Theta_{\tilde{\nu}}\right)_{1 j} \simeq 10^{-12}$ in order to satisfy correct freeze-in relic density. Following Eq. (5) (after neutrino mixing pattern is taken into account) one finds that,

$$
\left[Y_{\nu}\left(\left(A_{\nu}-\mu \cot \beta\right) \mathbb{I} \mp M_{N}\right)\right]_{i j} \simeq 10^{-8} \mathrm{GeV} \text { for } \mathrm{i}, \mathrm{j}=1,2,3,
$$

where $\mathbb{I}$ is $3 \times 3$ identity matrix.

In the absence of $\Delta L=2$ masses $\left(M_{N}\right)$, Eq. (15) is easily satisfied. In this case neutrino oscillation data fixes $Y_{\nu} \simeq 10^{-13}$, and hence one needs $\left(A_{\nu}-\mu \cot \beta\right) \leq$ $10^{5} \mathrm{GeV}$, which is consistent with $A_{\nu}$ and $\mu$ on the order of several hundreds of $\mathrm{GeV}$. On the other hand, the presence of $M_{N}$ further constrains $Y_{\nu}$, thus satisfying Eq. (15) for $\left(A_{\nu}-\mu \cot \beta\right)$ within a limited range. This allowed range depends on $M_{\tilde{\nu}_{\mathrm{DM}}^{i}}$, with larger values of $M_{\tilde{\nu}_{\mathrm{DM}}^{i}}$ allowing smaller $\left(A_{\nu}-\mu \cot \beta\right)$ following equation (14). In determining this allowed range we have taken into consideration the relic density of both the $C P$-even and $C P$-odd LSP states, i.e., in our notation $\Omega_{\mathrm{FI}} h^{2}=\Omega_{\tilde{\nu}_{\mathrm{DM}}^{1}} h^{2}+\Omega_{\tilde{\nu}_{\mathrm{DM}}^{2}} h^{2}$. Following the equation (15) it is also evident that the matrix $Y_{\nu} M_{N}$ is also tightly constrained. As already mentioned in Sec. III, the Yukawa couplings corresponding to lightest neutrino $\left[\left(Y_{\nu}\right)_{1 i}\right]$ have been chosen to be very small and hence the corresponding Majorana mass $\left(M_{N}\right)_{11}$ is not so tightly constrained as the other Majorana masses $\left(M_{N}\right)_{H}$. We kept $\left(M_{N}\right)_{11}$ fixed during our analysis. On the other hand, Majorana masses $\left(M_{N}^{H}\right)$ are allowed only up to a few tens of $\mathrm{GeV}$. In view of the BBN constraints on the lifetime of heavy neutrinos, we have considered two possible ranges of $M_{N}^{H}$ :

(1) In the first case we considered all the Majorana masses $\left[\left(M_{N}\right)_{11}, M_{N}^{H}\right]$ to be on the keV-scale and denote the Majorana mass matrix as $M_{N} \mathbb{I}$, where $M_{N}=\left(M_{N}\right)_{11}=M_{N}^{H}$.

(2) In the second case, the heavier Majorana masses $\left[\left(M_{N}\right)_{22,33}=M_{N}^{H}\right]$ have been assumed to be in the range $500 \mathrm{MeV}-20 \mathrm{GeV}$ while the lightest $\Delta L=2$ mass $\left(M_{N}\right)_{11}$ is kept fixed at $10 \mathrm{keV}$.

\section{1. keV scale Majorana mass}

The dependence of relic density on the supersymmetric parameters $\left[A_{\nu}, \mu,\left(m_{N}\right)_{11}\right]$ and Majorana $\operatorname{masses}\left(M_{N}\right)$ are shown in Fig. 1. As argued in Sec. III, the determining factor is the mixing angles $\Theta_{\tilde{\nu} 1 j}$ and hence the elements of the matrix $Y_{\nu}\left(A_{\nu}-\mu \cot \beta \mathbb{I} \mp M_{N} \mathbb{I}\right)$. For all the elements of the matrix $M_{N}$ are in the keV range, the largest element of the matrix $Y_{\nu}$ is $\simeq 10^{-10}$, leading to $M_{N}\left(Y_{\nu}\right)_{i j} \simeq$ $10^{-14} \mathrm{GeV}$ for all $\mathrm{i}, \mathrm{j}=1,2,3$, which is much smaller than $10^{-8} \mathrm{GeV}$ that is required for correct relic-density [see Eq. (15)]. Thus the correct relic density requires,

$$
\left(Y_{\nu}\right)_{i j}\left(A_{\nu}-\mu \cot \beta\right) \simeq 10^{-8} \mathrm{GeV} \text { for } \mathrm{i}, \mathrm{j}=1,2,3,
$$

which indicates $\left|A_{\nu}-\mu \cot \beta\right| \simeq \mathcal{O}(100 \mathrm{GeV})$ is allowed in order to obtain correct relic density for $M_{\tilde{\nu}_{\mathrm{DM}}^{i}} \simeq 100 \mathrm{GeV}$. One may wonder how is this situation different from the pure Dirac neutrino masses. One should remember that in case of pure Dirac masses all the elements of $Y_{\nu} \simeq \mathcal{O}\left(10^{-13}\right)$, while even the tiny Majorana masses considered here have raised some of the elements of $Y_{\nu}$ to $\mathcal{O}\left(10^{-10}\right)$ thereby affecting the allowed range of $\left|A_{\nu}-\mu \cot \beta\right|$. The Fig. 1(a) depicts how the relic density varies with $\mu$ for given values of $A_{\nu}$, while Fig. 1(b) shows the allowed region in $\mu-A_{\nu}$ plane for $\left(m_{N}\right)_{11} \simeq 100 \mathrm{GeV}$ and two different values of $M_{N}$. As $M_{N}$ increases from $10 \mathrm{keV}$ to $30 \mathrm{keV}$, the elements of $Y_{\nu} M_{N}$ in Eq. (15) increases and consequently the allowed region in $\mu-A_{\nu}$ plane shrinks. For each of these plots a minimum of relic density occurs when $\mu \cot \beta=A_{\nu}$. As $\mu$ departs from this 

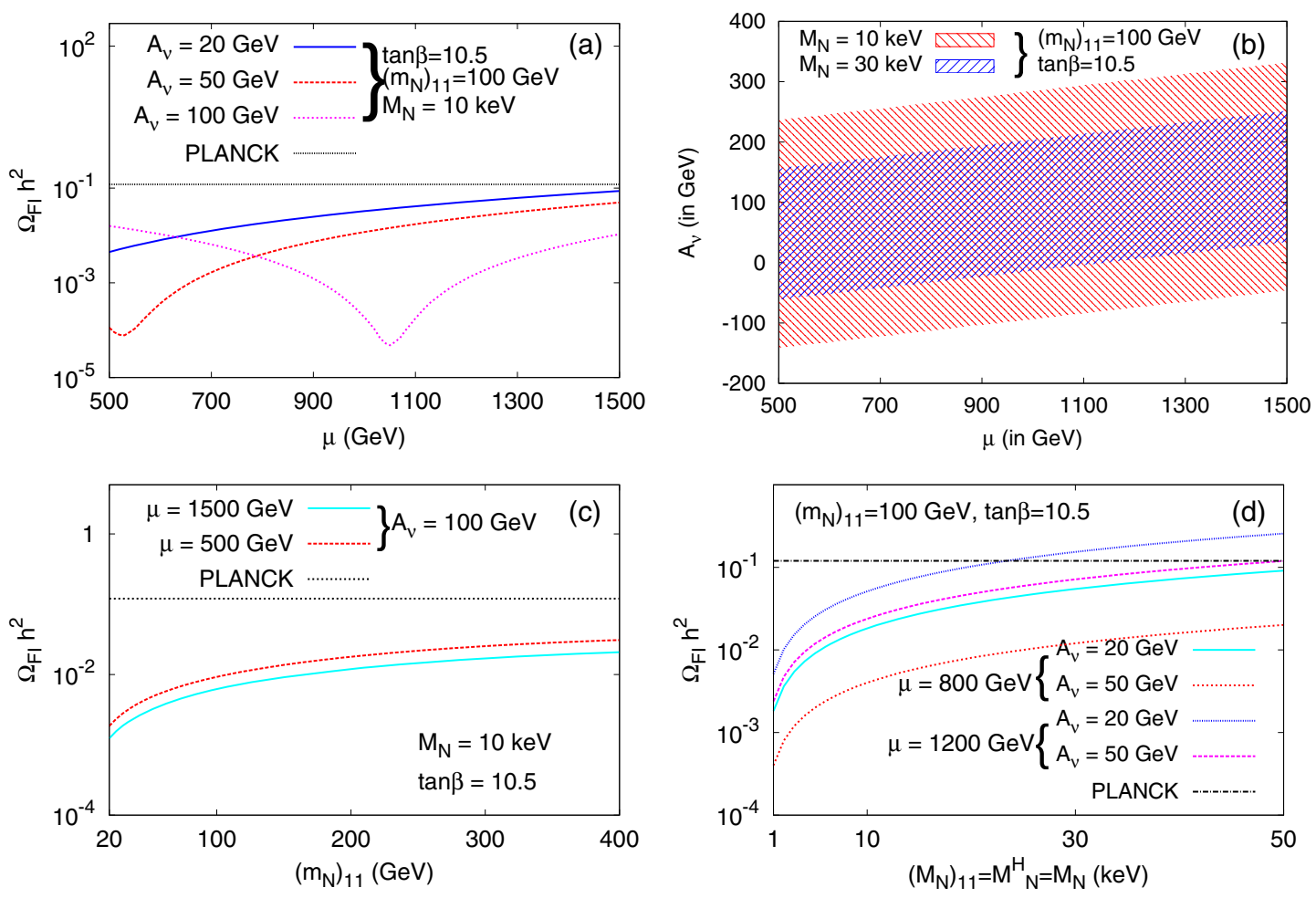

FIG. 1. For $m_{\text {susy }}=1 \mathrm{TeV}$ the dependence of freeze-in relic density with $\mu$ [subfigure 1(a)], $\left(m_{N}\right)_{11}$ [subfigure $1(\mathrm{c})$ ] and $M_{N}$ [subfigure 1(d)] have been shown. All the Majorana masses have been taken in the keV scale, $A_{\nu}$ is varied in the EW scale and $\tan \beta=10.5$ has been taken. The allowed region in the $\mu-A_{\nu}$ plane for $M_{N}=10 \mathrm{keV}$ is shown in the subfigure 1(b). This plot also embodies how the allowed region of $\mu-A_{\nu}$ plane shrinks as $M_{N}$ is increased.

point of exact cancellation relic density increases. In Figs. 1(c) and (d) we have shown the variation of relic density with $\left(m_{N}\right)_{11}$ and $M_{N}$ respectively. With increase in $\left(m_{N}\right)_{11}, M_{\tilde{\nu}_{\mathrm{DM}}^{i}}$ increases and relic density being proportional to $M_{\tilde{\nu}_{\mathrm{DM}}^{i}}$ [see Eq. (9)], also increases linearly with $\left(m_{N}\right)_{11}$. Whereas, with increase in $M_{N},\left(Y_{\nu}\right)_{i j}$ increases and relic increases pertaining to increase in $\Theta_{\tilde{\nu} 1 j}$.

Thus there is ample parameter space available for a rightsneutrino to be the LSP in presence of $M_{N} \simeq \mathcal{O}(\mathrm{keV})$. However, our choice of $\mathrm{keV}$-scale Majorana masses $\left(M_{N}\right)$ force all the sterile neutrinos to be warm DM. The Yukawa couplings $\left(Y_{\nu}\right)$, being always consistent with neutrino oscillation data, yield too large a relic density via DW mechanism for the corresponding heavy neutrinos [34] owing to their large mixing angles.

\section{Majorana masses in the $\mathrm{MeV}-\mathrm{GeV}$ range}

Since the range of $50 \mathrm{keV}-500 \mathrm{MeV}$ is disfavored by BBN constraints, we now focus on heavy neutrino Majorana masses $\left(M_{N}^{H}\right)$ in the $500 \mathrm{MeV}$ - a few tens of $\mathrm{GeV}$ range. The dependence of right sneutrino relic density on SUSY parameters $A_{\nu}, \mu,\left(m_{N}\right)_{11}$ and $M_{N}^{H}$ are shown in Fig. 2. The largest value of $\left(Y_{\nu}\right)_{i j}$ is $\simeq 10^{-8}$ for $M_{N}^{H} \simeq 500 \mathrm{MeV}$, implying the largest element of
$Y_{\nu} M_{N}^{H} \simeq 10^{-8} \mathrm{GeV}$. Thus one requires $\left|A_{\nu}-\mu \cot \beta\right| \simeq$ $1 \mathrm{GeV}$ in order to obtain the correct relic density via freeze-in. This cancellation is clear in Fig. 2(a) where for a given $\mu$, a very small range of $A_{\nu}$ is allowed in order to satisfy the relic density. The allowed parameter space in $\mu-A_{\nu}$ plane where relic density is below the observed value, is depicted in Fig. 2(b) with red and blue band for $M_{N}^{H}=500 \mathrm{MeV}$ and $5 \mathrm{GeV}$ respectively. A magnified version of the region $900 \mathrm{GeV} \leq \mu \leq 950 \mathrm{GeV}$ is shown in the inset of Fig. 2(b). Which clearly shows the decrease in the permissible values of $A_{\nu}$ for a given $\mu$ as one increase Majorana mass $\left(M_{N}^{H}\right)$ from $500 \mathrm{MeV}$ to $5 \mathrm{GeV}$. The dependence of relic density with $\left(m_{N}\right)_{11}$ and $M_{N}^{H}$ is shown in Fig. 2(c) where the contours depicts the value of relic density. The nature of the contours can be explained as follows. An increase in $\left(m_{N}\right)_{11}$ raises $M_{\tilde{\nu}_{\mathrm{DM}}^{i}}$, and the relic density, too, increases linearly. On the other hand, the freeze-in relic density increases with $M_{N}^{H}$, since $\left(Y_{\nu}\right)_{i j}$ (for $\mathrm{i}, \mathrm{j}=2,3)$ and hence $\Theta_{\tilde{\nu} 1 j}$ also increase with $M_{N}^{H}$. Thus as we increase either $\left(m_{N}\right)_{11}$ or $M_{N}^{H}$ the relic density increases. One should notice that even when a fine cancellation between $\mu \cot \beta$ and $A_{\nu}$ takes place (viz. in Fig. 2(c) $\left|A_{\nu}-\mu \cot \beta\right| \simeq 0.38 \mathrm{GeV}$ ), $M_{N}^{H}$ is allowed to be only up to a few tens of $G e V$, depending on the value of $\left(m_{N}\right)_{11}$. This is because, although $\left|A_{\nu}-\mu \cot \beta\right|$ is fixed at 

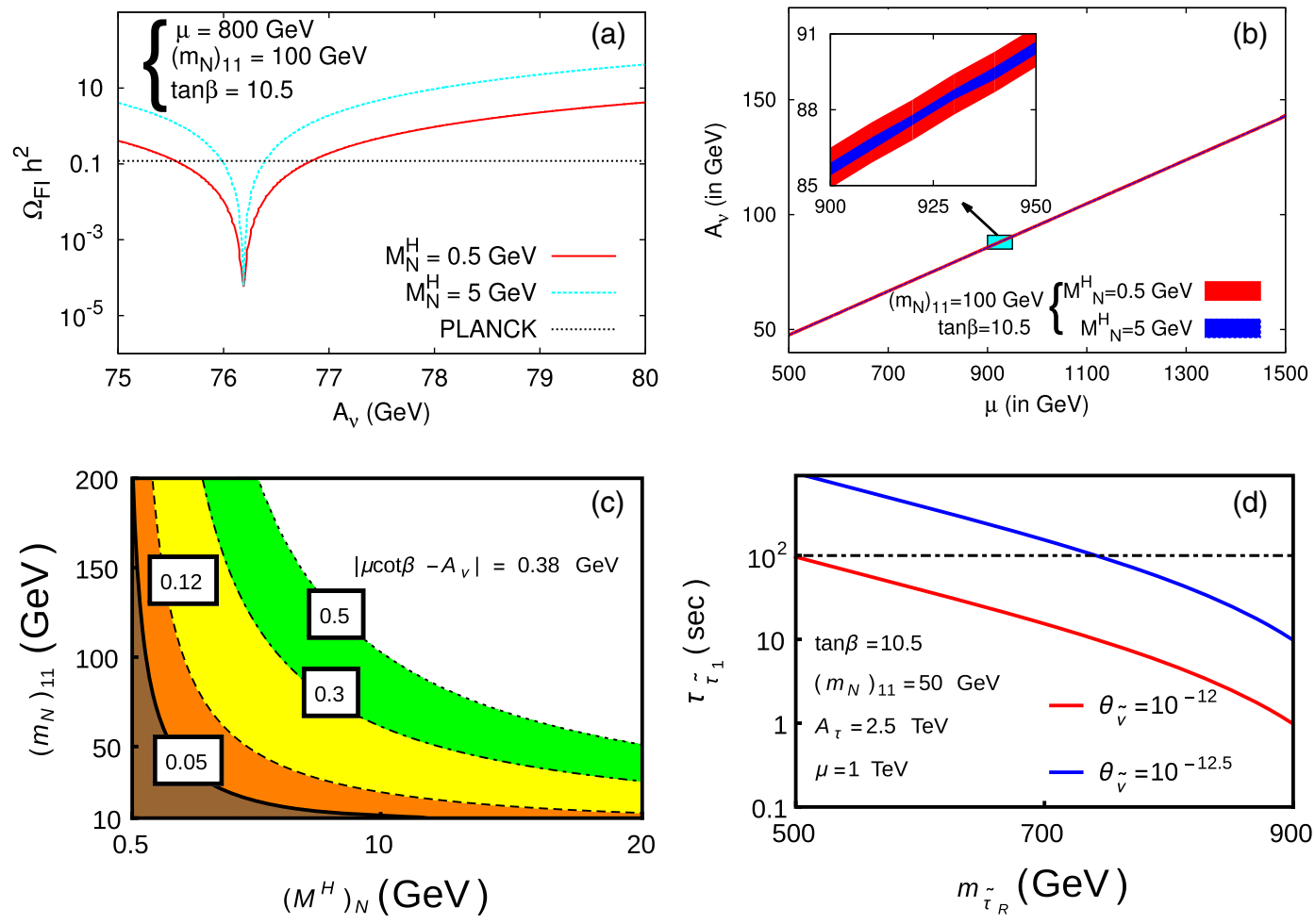

FIG. 2. Subfigure 2(a) shows how the exact cancellation between supersymmetry conserving term $\mu \cot \beta$ and supersymmetry breaking term $A_{\nu}$ leads to correct freeze-in relic density for sneutrino with MeV-GeV scale Majorana masses. Subfigures 2(b) and 2(c) show the variation of freeze-in relic density with $\left(m_{N}\right)_{11}$ and $M_{N}$ respectively. For each of the different values of $\mu, A_{\nu}$ has been chosen such that Eq. (15) is satisfied and one obtains the maximum possible values of $\left(m_{N}\right)_{11}\left(M_{N}\right)$ for that choice of $\left|A_{\nu}-\mu \cot \beta\right|$. If one chooses $A_{\nu}$ and $\mu \cot \beta$ to cancel more finely, one will get a larger value of $\left(m_{N}\right)_{11}\left(M_{N}\right)$, but the order of magnitude will not change. Subfigure 2(d) shows the allowed $\tilde{\tau}_{R}$ soft masses $m_{\tilde{\tau}_{R}}$ of $\tilde{\tau}_{1}$ NLSP such that its lifetime is $\leq 100$ sec. All the parameters $\left(A_{\tau}, \mu, \tan \beta, \theta_{\tilde{\nu}} \approx \Theta_{\tilde{\nu} 13}\right)$ that contributes to $\tilde{\tau}_{1}$ lifetime, are quoted in the figure. We have assumed $\tan \beta=10.5$ in all the cases.

$0.38 \mathrm{GeV},\left(Y_{\nu}\right)_{i j}$ (for $\left.\mathrm{i}, \mathrm{j}=2,3\right)$ increases with an increasing $M_{N}^{H}$ and consequently $\left(A_{\nu}-\mu \cot \beta \mp M_{N}^{H}\right)\left(Y_{\nu}\right)_{i j}$ becomes $\geq 10^{-8} \mathrm{GeV}$ for some value of $M_{N}^{H}$, thereby overclosing the universe.

A rather interesting signature of Dirac sneutrino LSP comes in the form of long-lived $\tilde{\tau}_{1}$ (lightest $\tilde{\tau}$ mass eigenstate, which is dominantly right-handed) NLSPs $[10,13,16,28]$. A $\tilde{\tau}_{1}$ NLSP decays to $\tilde{\nu}_{\mathrm{DM}}$ in association with a $W^{ \pm}$boson. The decay width is given by,

$$
\begin{aligned}
\Gamma_{\tilde{\tau}_{1}}= & \frac{g^{2} \Theta_{\tilde{\nu} 13}^{2}}{32 \pi}\left|U_{L 1}^{\left(\tilde{\tau}_{1}\right)}\right|^{2} \frac{M_{\tilde{\tau}_{1}}^{3}}{m_{W}^{2}}\left[1-\frac{2\left(M_{\tilde{\nu}_{\mathrm{DM}}}^{2}+m_{W}^{2}\right)}{M_{\tilde{\tau}_{1}}^{2}}\right. \\
& \left.+\frac{\left(M_{\tilde{\nu}_{\mathrm{DM}}}^{2}-m_{W}^{2}\right)^{2}}{M_{\tilde{\tau}_{1}}^{4}}\right]^{3 / 2},
\end{aligned}
$$

where $M_{\tilde{\tau}_{1}}$ is the $\tilde{\tau}_{1}$ mass, $m_{W}$ is the $W$-boson mass, and $U_{L 1}^{\tilde{\tau_{1}}}=\sin \theta_{\tilde{\tau}_{1}}$. Where, the stau mixing angle $\theta_{\tilde{\tau}_{1}}$ is given by,

$$
\tan 2 \theta_{\tilde{\tau}_{1}}=\frac{2 y_{\tau} v \sin \beta\left|A_{\tau}-\mu \cot \beta\right|}{m_{\tilde{l}_{L}}^{2}-m_{\tilde{\tau}_{R}}}
$$

All the parameters given in Eq. (18) are self-explanatory and their values can be found in Fig. 2(d). One may wonder whether a $\tilde{\tau}_{1}$ can be the NLSP in presence of $\Delta L=2$ masses, since the sneutrino mixing angles $\Theta_{\tilde{\nu} 1 j}$, which determines the lifetime of $\tilde{\tau}_{1}$, is severely constrained in the present scenario. Figure 2(d) shows the variation of $\tilde{\tau}_{1}$ lifetime with its mass when right-sneutrino has correct relic density, i.e., $\Theta_{\tilde{\imath} 1 j} \lesssim 10^{-12}$. Clearly a wide range of values of $M_{\tilde{\tau}_{1}}$ is allowed where the lifetime of $\tilde{\tau}_{1}$ is $\leq 100 \mathrm{sec}$, a constraint imposed by BBN. Thus even in presence of $\Delta L=2$ masses, right- sneutrino can serve as a nonthermal DM candidate along with a $\tilde{\tau}_{1}$ NLSP, that leaves its footprints in the form of a heavy stable charged track inside collider detectors.

\section{B. Results for $\boldsymbol{m}_{\text {susy }} \simeq \mathcal{O}(100) \mathrm{TeV}$}

As we have seen, for $m_{\text {susy }} \simeq 1 \mathrm{TeV}$, Majorana masses up to a few tens of $\mathrm{GeV}$ are allowed. If we allow even larger values for heavy neutrino Majorana masses $\left(M_{N}^{H}\right)$ then the Yukawa couplings also increase leading to an increase in the mixing angles $\Theta_{\tilde{\nu} 1 j}$. To compensate the effect we have to increase $m_{\text {susy }}$, and the denominator of the matrix $\Theta_{\tilde{\nu}}$ 

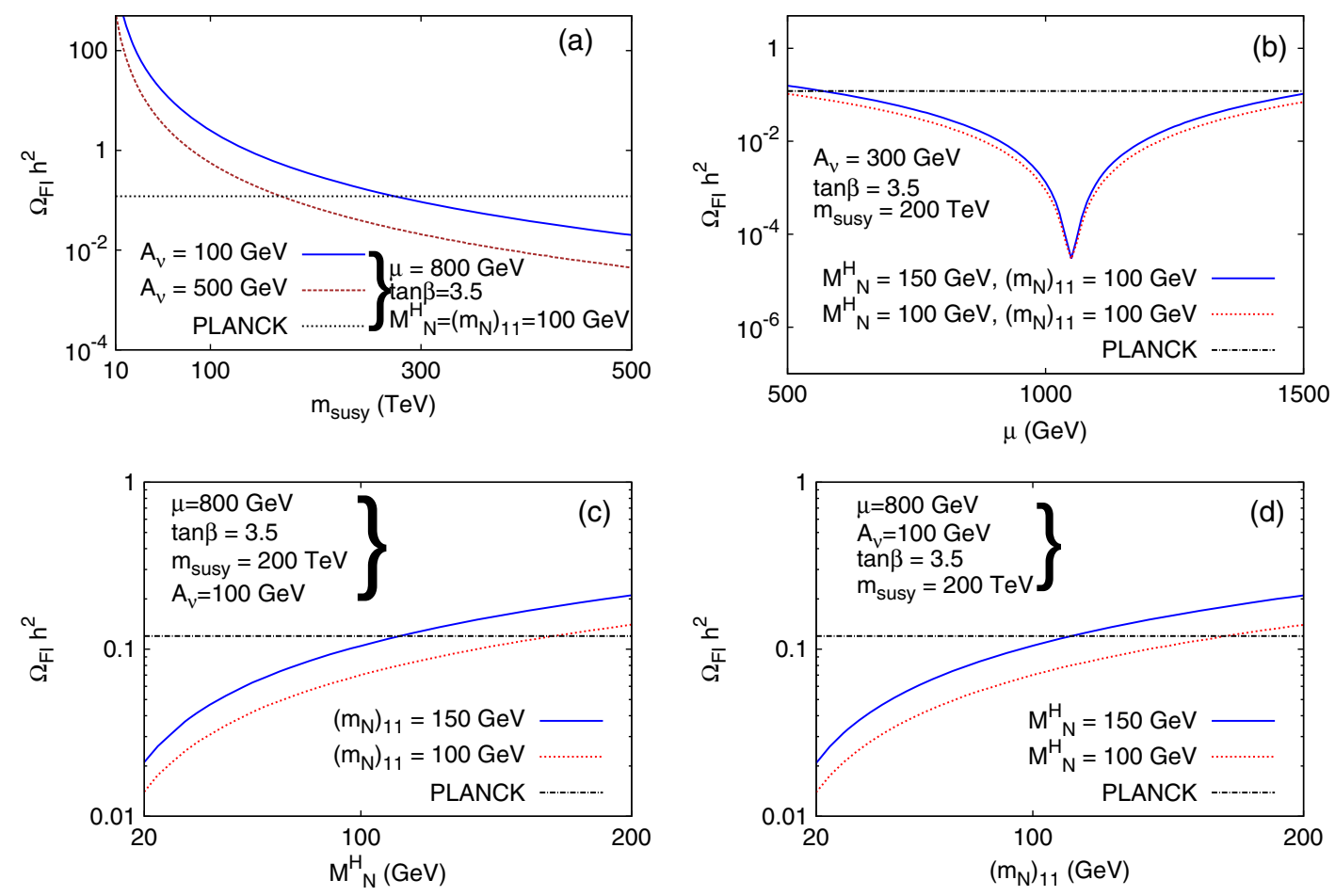

FIG. 3. Subfigure 3(a) show the variation of relic density a with increase in $m_{\text {susy }}$ for $A_{\nu}, \mu,\left(m_{N}\right)_{11}$, and $M_{N}^{H}$ around the electroweak scale. In order to generate correct SM like Higgs mass and satisfy all the constraints of EWSB $\tan \beta=3.5$ have been taken. Subfigure 3(b) depicts how the allowed region of $\mu-A_{\nu}$ plane have been extended due to such increase in SUSY-breaking masses i.e., $m_{\text {susy }}$. The subfigure 3(c) and 3(d) plots of the lower panel show the variation of freeze-in relic density with $\left(m_{N}\right)_{11}$ and $M_{N}^{H}$ respectively.

consequently increases. As an example, for superparticle masses around $100 \mathrm{TeV}$ (or beyond), the relic density requirement can be expressed as

$$
\sum_{i} M_{\tilde{\nu}_{\mathrm{DM}}^{i}} \Gamma_{\tilde{\nu}_{\mathrm{DM}}^{i}} \lesssim 5.2 \times 10^{-15} \mathrm{GeV}^{2}
$$

If one assumes the DM masses to be still in the electroweak scale, the above equation implies $\Theta_{\tilde{\nu} 1 j} \simeq 10^{-9}$. From Eq. (5) it is evident that $M_{N}^{H} \simeq 100 \mathrm{GeV}$ will be allowed in this scenario.

Figure 3(a) shows the dependence of relic density on $m_{\text {susy }}$ for $M_{N}^{H}=\left(m_{N}\right)_{11}=100 \mathrm{GeV}$. Clearly, for $m_{\text {susy }} \simeq 100 \mathrm{TeV}$, the relic density is satisfied depending on the value of $\left|A_{\nu}-\mu \cot \beta\right|$. Figure 3(b)depicts the dependence of relic density on $\mu$ (for a fixed $A_{\nu}$ and $\tan \beta$ ), which can differ from $A_{\nu} \tan \beta$ up to a few hundreds of $\mathrm{GeV}$ in this case. The dependency of relic density on $M_{N}^{H}$ and $\left(m_{N}\right)_{11}$ is shown in Figs. 3(c) and (d) respectively. Both the plots show the expected increase in relic density as we go on increasing $M_{N}^{H}$ and $\left(m_{N}\right)_{11}$. The explanation for the predicted behaviours can be found in the discussion of Fig. 2 in the previous subsection.

One should note that $\mu, M_{\tilde{\nu}_{\mathrm{DM}}^{i}}$, and $A_{\nu}$ have been varied around the electroweak scale with $\tan \beta=3.5$. This is because electroweak symmetry breaking conditions are difficult to satisfy with larger $\tan \beta$, if $m_{\text {susy }}$ is as high as $100 \mathrm{TeV}$. It is quite understandable that $M_{\tilde{\nu}_{\mathrm{DM}}^{i}}$ should be of $\mathcal{O}(100 \mathrm{GeV})$ in order to obtain correct relic density [following Fig. 3(d)]. On the other hand, $\mu$ should also be within a TeV, not only for satisfying electroweak symmetry breaking conditions, but also to control freeze-in via $\tilde{\nu}_{L} \rightarrow \tilde{\nu}_{R} h$. And consequently a $A_{\nu} \simeq$ $100 \mathrm{GeV}$ is also required so that $\left|A_{\nu}-\mu \cot \beta\right|$ does not become large enough to make the universe overclose. Hence, in this scenario we face an inexplicable hierarchy of SUSY-breaking parameters, to obtain the correct relic density, unless one engineer some highly contrived finetuning between $A_{\nu}$ and $\mu \cot \beta$ (see Sec. IVA 2).

\section{SUMMARY AND CONCLUSIONS}

We have worked with an MSSM scenario augmented with three families of right-chiral neutrino superfields, and $\Delta L=2$ mass terms in the superpotential as well as the scalar potential where masses for the light neutrinos have been generated following Type-I seesaw mechanism. The right-handed sneutrinos are nonthermal dark matter candidate as a result of small Yukawa couplings. We have restricted to hierarchical neutrino masses, latest constraints from Planck data on DM as well as data on the neutrino sector. We have gone beyond earlier studies [30] where constraints on the neutrino Majorana masses were obtained, 
mostly within a degenerate neutrino scenario. While the eV-scale scenario proposed there is presently disfavored, we have shown that the current picture admits of considerable varieties as well as constraints in view of current observations.

(i) While the lightest neutrino mass and consequently the corresponding entry in the (diagonal) righthanded neutrino mass matrix is a free parameter, the remaining $\Delta L=2$ mass terms are constrained rather tightly.

(ii) If all three $\Delta L=2$ entries in $M_{N}$ are in the $\mathrm{keV}$ range, there is an allowed region of parameter space to realize a right-handed sneutrino as nonthermal DM. But the all three sterile neutrinos, too, effectively constitute warm dark matter. Such a situation, however, is disfavored by the Dodelson-Widrow mechanism where the abundance of the warm DM goes up unacceptably.

(iii) For $\Delta L=2$ masses in the range $500 \mathrm{MeV}$-a few $\mathrm{GeV}$, all constraints can be satisfied only if $A_{\nu}$ and the $\mu$-parameter are considerably fine-tuned.

(iv) $\Delta L=2$ masses on the electroweak scale and above are possible only if DM mass $M_{\tilde{\nu}_{\mathrm{DM}}^{i}}, \mu$ and $A_{\nu}$ are 2-3 orders of magnitude smaller than the remaining
SUSY-breaking parameters, the later being on the order of $100 \mathrm{TeV}$.

We thus conclude that the right-sneutrino DM scenario, if at all the picture of nature, is subject to rather severe constraints in presence of the $\Delta L=2$ masses. The exception to this requires an inexplicable hierarchy among SUSY-breaking mass parameters or a severe cancellation between SUSYconserving $\mu$ and SUSY-breaking parameter $A_{\nu}$.

\section{ACKNOWLEDGMENTS}

We thank Raj Gandhi and Saptarshi Roy for helpful discussions. A. G. and B. M. acknowledges the hospitality of the Theoretical Physics Department, Indian Association for Cultivation of Science, Kolkata, where the last part of the project was carried out. This work was partially supported by funding available from the Department of Atomic Energy, Government of India, for the Regional Centre for Accelerator-based Particle Physics (RECAPP), Harish-Chandra Research Institute.

\section{APPENDIX A: DECAY WIDTHS}

The decay widths of the channels that contribute to freeze-in of $\tilde{\nu}_{\mathrm{DM}}$ are as follows [83]:

$$
\begin{gathered}
\Gamma\left(\tilde{H}_{u}^{0} \rightarrow \tilde{\nu}_{R} \nu_{L}\right)=\frac{1}{32 \pi M_{\tilde{H}_{u}^{0}}^{0}} \beta_{f}\left(M_{\tilde{H}_{u}^{0}}, M_{\nu_{L}}\right)\left(M_{\tilde{H}_{u}^{0}}^{2}+M_{\nu_{L}}^{2}-M_{\tilde{\nu}_{R}}^{2}\right)\left|\mathcal{A}\left(\tilde{H}_{u}^{0} \rightarrow \tilde{\nu}_{R} \nu_{L}\right)\right|^{2}, \\
\Gamma\left(\tilde{H}_{u}^{+} \rightarrow \tilde{\nu}_{R} l^{+}\right)=\frac{1}{32 \pi M_{\tilde{H}_{u}^{+}}} \beta_{f}\left(M_{\tilde{H}^{+}}, M_{l}\right)\left(M_{\tilde{H}_{u}^{+}}^{2}+M_{l}^{2}-M_{\tilde{\nu}_{R}}^{2}\right)\left|\mathcal{A}\left(\tilde{H}_{u}^{+} \rightarrow \tilde{\nu}_{R} l^{+}\right)\right|^{2}, \\
\Gamma\left(\tilde{\nu}_{L} \rightarrow \tilde{\nu}_{R} h\right)=\frac{1}{32 \pi M_{\tilde{\nu}_{L}}} \beta_{f}\left(M_{\tilde{\nu}_{L}}, M_{h}\right)\left|\mathcal{A}\left(\tilde{\nu}_{L} \rightarrow \tilde{\nu}_{R} h\right)\right|^{2}, \\
\Gamma\left(\tilde{\nu}_{L} \rightarrow \tilde{\nu}_{R} Z\right)=\frac{1}{32 \pi M_{\tilde{\nu}_{L}}} \frac{M_{\tilde{\nu}_{L}}^{4}}{M_{Z}^{2}} \beta_{f}^{3}\left(M_{\tilde{\nu}_{L}}, M_{Z}\right)\left|\mathcal{A}\left(\tilde{\nu}_{L} \rightarrow \tilde{\nu}_{R} Z\right)\right|^{2}, \\
\Gamma\left(\tilde{l}_{L} \rightarrow \tilde{\nu}_{R} W^{-}\right)=\frac{1}{32 \pi M_{\tilde{l}_{L}}} \frac{M_{\tilde{l}_{L}}^{4}}{M_{W}^{2}} \beta_{f}^{3}\left(M_{\tilde{l}_{L}}, M_{W}\right)\left|\mathcal{A}\left(\tilde{\nu}_{L} \rightarrow \tilde{\nu}_{R} L^{-}\right)\right|^{2}, \\
\Gamma\left(\tilde{W}^{0} \rightarrow \tilde{\nu}_{R} \nu_{L}\right)=\frac{1}{32 \pi M_{\tilde{B}^{0}}} \beta_{f}\left(M_{\tilde{B}^{0}}, M_{\nu_{L}}\right)\left(M_{\tilde{B}^{0}}^{2}+M_{\nu_{L}}^{2}-M_{\tilde{\nu}_{R}}^{2}\right)\left|\mathcal{A}\left(\tilde{B}^{0} \rightarrow \tilde{\nu}_{R} \nu_{L}\right)\right|^{2}, \\
\Gamma\left(\tilde{W}^{+} \rightarrow \tilde{\nu}_{R} l^{+}\right)=\frac{1}{32 \pi M_{\tilde{W}^{+}}} \beta_{f}\left(M_{\tilde{W}^{0}}, M_{\nu_{L}}\right)\left(M_{\tilde{W}^{+}}^{2}, M_{l}\right)\left(M_{\tilde{W}^{+}}^{2}+M_{\nu_{L}}^{2}-M_{\tilde{\nu}_{R}}^{2}\right)\left|\mathcal{A}\left(\tilde{W}^{0} \rightarrow \tilde{\nu}_{R} \nu_{L}\right)\right|^{2},
\end{gathered}
$$

where $\beta_{f}\left(M_{A}, M_{B}\right)=\left(1-2 \frac{M_{\tilde{\nu}_{R}}^{2}+M_{B}^{2}}{M_{A}^{2}}+\frac{\left(M_{\tilde{\nu}_{R}}^{2}-M_{B}^{2}\right)^{2}}{M_{A}^{4}}\right)^{1 / 2}$ and $\mathcal{A}_{A \rightarrow B \tilde{\nu}_{R}}$ is the amplitude for the decay $A \rightarrow B \tilde{\nu}_{R}$. We have tabulated all the relevant amplitudes in B. 


\section{APPENDIX B: AMPLITUDES}

In this section we give the expressions for the amplitudes for all the decays considered for calculation of freezein relic.

(1) The two blocks of the sneutrino mass-matrix $M_{\tilde{\nu}}$ given in equation (4) are diagonalized by two different hermitian matrices. We denote both of these matrices as $Z_{\tilde{\nu}}$ and use the appropriate matrix depending on whether we are calculating the relic density of $C P$-even or $C P$-odd $\tilde{\nu}_{R}$ LSP. It is quite clear that there being $6 C P$-even and $6 C P$-odd sneutrinos each of these $Z_{\tilde{\nu}}$ are $6 \times 6$ matrices which one can cast in terms of the sneutrino mixing matrix $\Theta_{\tilde{\nu}}$ as,

$$
Z_{\tilde{\nu}} \approx\left[\begin{array}{cc}
\mathbb{I} & -\Theta_{\tilde{\nu}}^{T} \\
\Theta_{\tilde{\nu}} & \mathbb{I}
\end{array}\right]
$$

where $\mathbb{I}$ is $3 \times 3$ identity matrix. The mixing of the lightest sneutrino eigenstate with the left-handed flavor eigenstate sneutrinos are given by $Z_{\tilde{\nu} 4 k}=$ $\Theta_{\tilde{\nu} 1 k}$ with $\mathrm{k}=1,2,3$ and the corresponding mixing with right-chiral sneutrinos are given by $Z_{\tilde{\nu} 4(3+k)}=$ $\delta_{1 k}$ where $\mathrm{k}=1,2,3$.

(2) The full $6 \times 6$ mass-matrices of neutrinos $\left(\nu_{L i}, N_{R i}\right.$ with $i=1,2,3)$ is given by,

$$
M_{\nu}=\left[\begin{array}{cc}
0 & m_{D}^{T} \\
m_{D} & M_{N}
\end{array}\right]
$$

which is diagonalized by a $6 \times 6$ matrix,

$$
U_{V}=\left[\begin{array}{cc}
U_{0} & S \\
S & U_{H}
\end{array}\right]
$$

where the top $3 \times 3$ block denoted by $U_{0}$ is the usual PMNS-matrix. $S$ denotes the mixing between $\nu_{L} \mathrm{~S}$ and $N_{R} \mathrm{~s}$ and $U_{H}$ denotes the mixing between different generations of $N_{R}$ s.

In the expressions that follows among the free indices $i, j$ denotes the sneutrino generation appearing in the vertex while $I$ denotes the same for R-parity even particles and sleptons. Thus $i, j=1, \ldots, 6$ and $I=1,2,3$ are allowed. Where index $i=4$ corresponds to sneutrino LSP. The indices which has to be summed over have been shown explicitly. $P_{L}=\frac{1-\gamma_{5}}{2}$ is the left-handed projection operator, $g_{2}$ being the weak coupling of SM and $\theta_{w}$ is the Wienberg angle.

\section{Interactions with gauginos}

(1) $\mathcal{A}\left(\tilde{B}^{0} \rightarrow \tilde{\nu}_{R}^{i} \nu_{L}^{I}\right)=\frac{i}{2} g_{1} P_{L} \sum_{k=1}^{3} U_{0 I k}^{*} Z_{\tilde{\nu} i k}$
(2) $\mathcal{A}\left(\tilde{W}^{0} \rightarrow \tilde{\nu}_{R}^{i} \nu_{L}^{I}\right)=-\frac{i}{2} g_{2} P_{L} \sum_{k=1}^{3} U_{0 I k}^{*} Z_{\tilde{\nu} i k}$

(3) $\mathcal{A}\left(\tilde{H}_{u}^{0} \rightarrow \tilde{\nu}_{R}^{i} \nu_{L}^{I}\right)=-\frac{i}{\sqrt{2}} P_{L}\left(\sum_{k, l=1}^{3} Z_{\tilde{\nu} i k}^{*} Y_{\nu l k} U_{V I(3+l)}+\right.$ $\left.\sum_{k, l=1}^{3} Z_{\tilde{\nu} i(3+l)}^{*} Y_{\nu l k} U_{V I k}\right)$

(4) $\mathcal{A}\left(\tilde{W}^{-} \rightarrow \tilde{\nu}_{R}^{i} l^{-I}\right)=-\frac{i}{2} g_{2} P_{L} Z_{\tilde{\nu} i I}^{*}$

(5) $\mathcal{A}\left(\tilde{H}_{u}^{+} \rightarrow \tilde{\nu}_{R}^{i} l^{-I}\right)=\frac{i}{2} P_{L} \sum_{k=1}^{3} Z_{\tilde{\nu} i(3+k)}^{*} Y_{\nu k I}$

\section{Interactions with sfermions}

Sneutrino LSP can decay from sleptons and heavier sneutrinos via SM gauge bosons or SM Higgs which we have discussed below:

\section{a. Interactions with gauge-bosons}

(1) $\mathcal{A}\left(\tilde{e}_{L}^{I} \rightarrow \tilde{\nu}_{R}^{i} W^{\mu}\right)=\frac{i}{2} g_{2} Z_{\tilde{\nu} i I}^{*}\left(p_{\tilde{\nu}^{i}}-p_{\tilde{e}_{L}}\right)^{\mu}$

(2) $\begin{aligned} \mathcal{A}\left(\tilde{\nu}_{H}^{j} \rightarrow \tilde{\nu}_{R}^{i} Z^{\mu}\right)= & \frac{1}{2}\left(g_{2} \cos \theta_{w}+g_{1} \sin \theta_{w}\right)\left(p_{\tilde{\nu}_{H}}-p_{\tilde{\nu}^{i}}\right)^{\mu} \times \\ & \sum_{k=1}^{3} Z_{\tilde{\nu} j k}^{* H} Z_{\tilde{\nu} i k}^{*},\end{aligned}$ here $H$ denotes the heavier sneutrino eigenstates and hence $\mathrm{j}$ runs from 1 to 5 . One has to keep in mind that this vertex is non-zero only when the $C P$ nature of the two participating sneutrinos $\left(\tilde{\nu}_{H}^{j}\right.$ and $\left.\tilde{\nu}^{i}\right)$ are opposite. Hence if $Z_{\tilde{\nu}}$ is the diagonalizing matrix corresponding to $C P$-even states then $Z_{\tilde{\nu}}^{H}$ has to be that corresponding to $C P$-odd states.

\section{b. Interactions with SM Higgs}

(1) For a heavier $C P$-even(or $C P$-odd) $\operatorname{sneutrino}\left(\tilde{\nu}_{H}^{j}\right)$ decaying into a lighter $C P$-even(or $C P$-odd) sneutrino $\left(\tilde{\nu}^{i}\right)$,

$$
\begin{aligned}
\mathcal{A}\left(\tilde{\nu}_{H}^{j}\right. & \left.\rightarrow \tilde{\nu}_{R}^{i} h\right) \\
= & \frac{i}{2 \sqrt{2}}\left(\mu \sum_{p, k=1}^{3}\left[Z_{\tilde{\nu} j k}^{* H} Z_{\tilde{\nu} i(3+p)}^{*}+Z_{\tilde{\nu} j(3+p)}^{* H} Z_{\tilde{\nu} i k}^{*}\right] Y_{\nu p k}^{*}\right. \\
& \left.+\mu^{*} \sum_{p, k=1}^{3}\left[Z_{\tilde{\nu} j k}^{* H} Z_{\tilde{\nu} i(3+p)}^{*}+Z_{\tilde{\nu} j(3+p)}^{* H} Z_{\tilde{\nu} i k}^{*}\right] Y_{\nu p k}\right) \\
& -2\left(g_{1}^{2}+g_{1}^{2}\right) v_{d} \sum_{k=1}^{3} Z_{\tilde{\nu} j k}^{* H} Z_{\tilde{\nu} i k}^{*}
\end{aligned}
$$

For Higgs-mediated sneutrino decays also $\mathrm{j}$ runs from 1 to 5 as in the case of Z-mediated processes and in this case both $Z_{\tilde{\nu}}$ and $Z_{\tilde{\nu}}^{H}$ have to be the mixing matrix corresponding to $C P$-even (or $C P$ odd) states.

In diagonalizing sneutrino mass matrix and calculating all the Feynman Rules we have used SARAH [84]. 
[1] G. Arcadi, L. Covi, and M. Nardecchia, Phys. Rev. D 92, 115006 (2015).

[2] A. Arbey, M. Battaglia, L. Covi, J. Hasenkamp, and F. Mahmoudi, Phys. Rev. D 92, 115008 (2015).

[3] R. Catena, L. Covi, and T. Emken, Phys. Rev. D 91, 123524 (2015).

[4] L. Covi, J. Phys. Conf. Ser. 485, 012002 (2014).

[5] L. Covi, in Proceedings of the 2nd Workshop on Flavor Symmetries and Consequences in Accelerators and Cosmology, FLASY 12, Dortmund, Germany, 2012 (2012), pp. 43-50, http://inspirehep.net/record/1192942.

[6] L. Covi, J. Hasenkamp, S. Pokorski, and J. Roberts, J. High Energy Phys. 11 (2009) 003.

[7] J. Dutta, P. Konar, S. Mondal, B. Mukhopadhyaya, and S. K. Rai, J. High Energy Phys. 09 (2017) 026.

[8] A. Ferrantelli, Eur. Phys. J. C 77, 716 (2017).

[9] C. Munoz, EPJ Web Conf. 136, 01002 (2017).

[10] S. Banerjee, G. Bélanger, A. Ghosh, and B. Mukhopadhyaya, J. High Energy Phys. 09 (2018) 143.

[11] L. Delle Rose, S. Khalil, S. J. D. King, S. Kulkarni, C. Marzo, S. Moretti, and C. S. Un, arXiv:1804.07753.

[12] A. Chatterjee, J. Dutta, and S. K. Rai, J. High Energy Phys. 06 (2018) 042.

[13] S. Banerjee, G. Bélanger, B. Mukhopadhyaya, and P. D. Serpico, J. High Energy Phys. 07 (2016) 095.

[14] C. Arina, M. E. C. Catalan, S. Kraml, S. Kulkarni, and U. Laa, J. High Energy Phys. 05 (2015) 142.

[15] M. E. Cabrera Catalan, Nucl. Part. Phys. Proc. 267-269, 356 (2015).

[16] W. Abdallah, A. Hammad, A. Kasem, and S. Khalil, Phys. Rev. D 98, 095019 (2018).

[17] S. Banerjee, P. S. B. Dev, S. Mondal, B. Mukhopadhyaya, and S. Roy, J. High Energy Phys. 10 (2013) 221.

[18] S. Biswas and B. Mukhopadhyaya, Phys. Rev. D 81, 015003 (2010).

[19] S. Biswas and B. Mukhopadhyaya, Phys. Rev. D 79, 115009 (2009).

[20] S. Biswas, Phys. Rev. D 82, 075020 (2010).

[21] D. Choudhury, S. K. Gupta, and B. Mukhopadhyaya, Phys. Rev. D 78, 015023 (2008).

[22] S. K. Gupta, B. Mukhopadhyaya, and S. K. Rai, Phys. Rev. D 75, 075007 (2007).

[23] T. Asaka, K. Ishiwata, and T. Moroi, Phys. Rev. D 73, 051301 (2006).

[24] T. Asaka, K. Ishiwata, and T. Moroi, Phys. Rev. D 75, 065001 (2007).

[25] T. Asaka, K. Ishiwata, and T. Moroi, AIP Conf. Proc. 903, 16 (2007).

[26] E. Molinaro, C. E. Yaguna, and O. Zapata, J. Cosmol. Astropart. Phys. 07 (2014) 015.

[27] A. G. Hessler, A. Ibarra, E. Molinaro, and S. Vogl, J. High Energy Phys. 01 (2017) 100.

[28] A. Ghosh, T. Mondal, and B. Mukhopadhyaya, J. High Energy Phys. 12 (2017) 136.

[29] D. Borah and A. Gupta, Phys. Rev. D 96, 115012 (2017).

[30] S. Gopalakrishna, A. de Gouvea, and W. Porod, J. Cosmol. Astropart. Phys. 05 (2006) 005.

[31] C. E. Yaguna, Phys. Lett. B 669, 139 (2008).

[32] I. Esteban, M. C. Gonzalez-Garcia, M. Maltoni, I. MartinezSoler, and T. Schwetz, J. High Energy Phys. 01 (2017) 087.
[33] S. Dodelson and L. M. Widrow, Phys. Rev. Lett. 72, 17 (1994).

[34] B. Shakya, Mod. Phys. Lett. A 31, 1630005 (2016).

[35] A. Boyarsky, O. Ruchayskiy, and D. Iakubovskyi, J. Cosmol. Astropart. Phys. 03 (2009) 005.

[36] D. Gorbunov, A. Khmelnitsky, and V. Rubakov, J. Cosmol. Astropart. Phys. 10 (2008) 041.

[37] R. Essig, E. Kuflik, S. D. McDermott, T. Volansky, and K. M. Zurek, J. High Energy Phys. 11 (2013) 193.

[38] A. Roy and M. Shaposhnikov, Phys. Rev. D 82, 056014 (2010).

[39] L. Canetti and M. Shaposhnikov, J. Cosmol. Astropart. Phys. 09 (2010) 001.

[40] T. Asaka, M. Laine, and M. Shaposhnikov, J. High Energy Phys. 01 (2007) 091; 02 (2015) 28.

[41] P. A. R. Ade et al. (Planck Collaboration), Astron. Astrophys. 594, A13 (2016).

[42] A. de Gouvea, Phys. Rev. D 72, 033005 (2005).

[43] R. H. Cyburt, B. D. Fields, K. A. Olive, and T.-H. Yeh, Rev. Mod. Phys. 88, 015004 (2016).

[44] M. Hirsch, H. V. Klapdor-Kleingrothaus, and S. G. Kovalenko, Phys. Lett. B 398, 311 (1997).

[45] L. J. Hall, T. Moroi, and H. Murayama, Phys. Lett. B 424, 305 (1998).

[46] Y. Grossman and H. E. Haber, Phys. Rev. Lett. 78, 3438 (1997).

[47] J. L. Feng, A. Rajaraman, and F. Takayama, Phys. Rev. Lett. 91, 011302 (2003).

[48] J. L. Feng, A. Rajaraman, B. T. Smith, S. Su, and F. Takayama, arXiv:hep-ph/0410178.

[49] L. J. Hall, K. Jedamzik, J. March-Russell, and S. M. West, J. High Energy Phys. 03 (2010) 080.

[50] L. Visinelli and P. Gondolo, Phys. Rev. D 91, 083526 (2015).

[51] I. R. Waldstein and A. L. Erickcek, Phys. Rev. D 95, 088301 (2017).

[52] L. Visinelli, Symmetry 10, 546 (2018).

[53] N. Bernal, M. Heikinheimo, T. Tenkanen, K. Tuominen, and V. Vaskonen, Int. J. Mod. Phys. A 32, 1730023 (2017).

[54] F. D'Eramo, N. Fernandez, and S. Profumo, J. Cosmol. Astropart. Phys. 02 (2018) 046.

[55] M. Becker, arXiv:1806.08579.

[56] A. Biswas, D. Borah, and D. Nanda, J. Cosmol. Astropart. Phys. 09 (2018) 014.

[57] D. Borah, B. Karmakar, and D. Nanda, J. Cosmol. Astropart. Phys. 07 (2018) 039.

[58] A. Biswas, D. Borah, and A. Dasgupta, arXiv:1805.06903.

[59] P. Gondolo and J. Edsjo, Nucl. Phys. B, Proc. Suppl. 70, 120 (1999); 70, 120 (1999).

[60] E. W. Kolb and M. S. Turner, Front. Phys. 69, 1 (1990).

[61] R. Adhikari et al., J. Cosmol. Astropart. Phys. 01 (2017) 025.

[62] M. H. Chan, Astrophys. Space Sci. 361, 116 (2016).

[63] F. Hofmann, J. S. Sanders, K. Nandra, N. Clerc, and M. Gaspari, Astron. Astrophys. 592, A112 (2016).

[64] K. Perez, K. C. Y. Ng, J. F. Beacom, C. Hersh, S. Horiuchi, and R. Krivonos, Phys. Rev. D 95, 123002 (2017).

[65] J. Baur, N. Palanque-Delabrouille, C. Yeche, A. Boyarsky, O. Ruchayskiy, E. Armengaud, and J. Lesgourgues, J. Cosmol. Astropart. Phys. 12 (2017) 013.

[66] C. Yèche, N. Palanque-Delabrouille, J. Baur, and H. du Mas des Bourboux, J. Cosmol. Astropart. Phys. 06 (2017) 047. 
[67] A. Schneider, J. Cosmol. Astropart. Phys. 04 (2016) 059.

[68] V. Poulin and P. D. Serpico, Phys. Rev. D 91, 103007 (2015).

[69] M. Kawasaki, K. Kohri, and T. Moroi, Phys. Rev. D 71, 083502 (2005).

[70] K. Jedamzik, Phys. Rev. D 74, 103509 (2006).

[71] F. Iocco, G. Mangano, G. Miele, O. Pisanti, and P. D. Serpico, Phys. Rep. 472, 1 (2009).

[72] K. Ishiwata, M. Kawasaki, K. Kohri, and T. Moroi, Phys. Lett. B 689, 163 (2010).

[73] W. Porod and F. Staub, Comput. Phys. Commun. 183, 2458 (2012).

[74] M. Aaboud et al. (ATLAS Collaboration), Phys. Lett. B 784, 345 (2018).

[75] G. Aad et al. (ATLAS and CMS Collaborations), Phys. Rev. Lett. 114, 191803 (2015).
[76] V. Khachatryan et al. (CMS Collaboration), Eur. Phys. J. C 75, 212 (2015).

[77] G. Aad et al. (ATLAS Collaboration), Eur. Phys. J. C 76, 6 (2016).

[78] J. Bernon and B. Dumont, Eur. Phys. J. C 75, 440 (2015).

[79] P. Bechtle, O. Brein, S. Heinemeyer, O. Stål, T. Stefaniak, G. Weiglein, and K. E. Williams, Eur. Phys. J. C 74, 2693 (2014).

[80] S. Antusch and O. Fischer, J. High Energy Phys. 10 (2014) 094.

[81] J. A. Casas and A. Ibarra,Nucl. Phys. B618, 171 (2001).

[82] A. Abada, S. Davidson, A. Ibarra, F. X. Josse-Michaux, M. Losada, and A. Riotto,J. High Energy Phys. 09 (2006) 010.

[83] H. Baer and X. Tata, Weak Scale Supersymmetry: From Superfields to Scattering Events (Cambridge University Press, Cambridge, England, 2006).

[84] F. Staub, Comput. Phys. Commun. 185, 1773 (2014). 